\title{
Economic Burden of Scorpion Sting and Snake Bite from a Social Perspective in Iran
}

\author{
Iman Mashhadi,, Zahra Kavousi, ${ }^{1}$ Payam Peymani,, Shokrollah Salman Zadeh Ramhormozi, ${ }^{3}$ and \\ Khosro Keshavarz, ${ }^{*}$ \\ ${ }^{1}$ Health Human Resources Research Center, School of Management and Information Sciences, Shiraz University of Medical Sciences, Shiraz, Iran \\ ${ }^{2}$ Health Policy Research Center, Shiraz University of Medical Sciences, Shiraz, Iran \\ ${ }^{3}$ Department of Infectious Diseases and Tropical Medicine, Faculty of Medicine, Ahvaz University of Medical Sciences, Ahvaz, Iran \\ "Corresponding author: Khosro Keshavarz, Health Human Resources Research Center, School of Management and Information Sciences, Shiraz University of Medical Sciences, \\ Shiraz, Iran. Tel: +98-9173301430, E-mail: khkeshavarz2007@gmail.com
}

Received 2017 January 16; Revised 2017 February 28; Accepted 2017 April 26.

\begin{abstract}
Background: Iran is one of the countries with a high incidence of venomous animal bites, especially scorpion stings and snake bites. As a result, paying inadequate attention to education and prevention programs can impose considerable economic and social costs on the country. Thus, this study aimed to determine the economic burden of snake bite and scorpion sting through identifying direct and indirect costs imposed on the patients in the country.

Methods: This was an applied, cross-sectional and analytical-descriptive study conducted in Ahvaz, Iran, in 2015. In order to collect data on direct medical costs, we used the data available in patients' medical records and self-reports of specialists. Data on nonmedical direct costs and indirect costs were collected through face-to-face or telephone interviews with the patients in the studied centers. Human capital approach was used to calculate indirect costs.

Results: The total annual costs estimated for the victims of scorpion sting and snake bite were 50,656,424 PPP\$ and 11,317,416 PPP\$, respectively. Of the mentioned costs, respectively, $90.6 \%$ and $75.1 \%$ were spent on direct costs and $9.4 \%$ and $24.9 \%$ on indirect costs. Conclusions: The results of this study showed that scorpion sting and snake bites could impose a significant economic burden on payers, patients, and the community. Therefore, it is necessary to adopt proper preventive and medical measures to prevent the increase of the related economic burden.
\end{abstract}

Keywords: Scorpion Sting, Snake Bite, Cost of Illness, Economic Burden

\section{Background}

In recent decades, health expenditure has increased considerably all over the world (1-3 ). In addition, because of the catastrophic costs of health-related services, the health system in Iran has been faced significant challenges $(4,5)$. The costs may be imposed directly by the use of diagnostic and medical services or indirectly by the loss of productivity and quality of life (6). According to recent reports by the World Health Organization, more than 5 million cases of snake bites, scorpion sting, wasp sting, etc. occur in the world each year that ultimately lead to 100 thousand deaths per year (7). In addition, most cases of snake bite occur in Asian countries leading to 30 thousand deaths annually on this continent. Moreover, at present in some parts of the world, including South Africa, Middle East, South American states including Mexico, and South America, and the Indian subcontinent, scorpion sting is one of the most important health problems (8). Real statistical data on scorpion sting are not available; however, the estimated annual number of cases worldwide is one million cases.
According to the reports, Mexico alone has 250,000 cases of scorpion sting and Tunisia has 40,000 cases of scorpion sting, of which 100 cases lead to patients' death (9). A total of 250,000 cases of venomous animal bites are annually reported in Islamic Republic of Iran; thus, Iran is among the countries with a high incidence of this type of injury (7). Deaths from bites occur in all regions of the country; however, about $75 \%$ of deaths occur in the three provinces of Khuzestan, Sistan and Baluchestan, and Kerman (7). Scorpion sting occurs in many parts of Iran; as a consequence, more than 100,000 people annually are injured by scorpion sting, of which more than $75 \%$ are children. Mortality from scorpion sting is 80 to 100 deaths per year, most of which occur in Khuzestan province $(10,11)$.

In addition, based on the annual estimates, approximately 300,000 cases of snake bite annually occur worldwide. Moreover, every year about 30000 - 40000 deaths due to snake bites are reported (12). Of all, 98\% of snake bites occur in the extremities (hands and feet) and 35\% of victims are children under 10 years of age. Furthermore, snake bite is more common in males (12). There are more 
than 3,000 kinds of snakes around the world that almost 350 kinds are venomous and of these, 150 to 180 kinds are among the dangerous venomous species (12). For instance, cobra snake bites can lead to the death of $25 \%$ of the victims (7). Undoubtedly, of all types of bites and accidents caused by venomous animals, bites of venomous snakes are among the most important causes of injuries and deaths in many parts of the world including Iran. Due to the geographic and climatic conditions and the presence of known types of venomous snakes in Iran, many injuries caused by snake bites occur every year in different regions of the country (12). So far, no study has been conducted and published on the economic burden of scorpion sting and snake bite in Iran. In addition, a few studies on this topic have been conducted and published in other parts of the world, as well. For instance, Edward et al.'s study (2013) evaluated the cost effectiveness of antiscorpion sting serum sold in the United States market and the results showed that the average cost of treatment using anti-scorpion sting serum was $10,708 \$$. The researchers concluded that anti-scorpion sting serum offered at current price in the US market is not cost-effective. They found that anti-scorpion serum available in the market is very effective but it is too costly (13).

Therefore, due to the lack of sufficient scientific evidence, the economic burden of scorpion sting and snake bites on community and individuals in the country is unclear. Because of special side effects of this type of injury, in the absence of follow-up and treatment services, it can impose a very high cost on the health system and the community. Moreover, it can impose a significant economic burden on patients and health systems and communities. Therefore, the economic burden of this type of injury can be very significant.

\section{Objectives}

The aim of this study was to estimate the direct and indirect costs of snake bite and scorpion sting in the country. As a result, providing detailed data on economic effects of scorpion sting and snake bite can help health policy makers in the country make informed decisions about the prevention and early treatment of the injury.

\section{Methods}

This was an applied, cross-sectional and analyticaldescriptive study conducted in Ahvaz, Iran, in 2015. It was carried out on patients suffering from scorpion sting and snake bite who referred to Abuzar and Razi hospitals in Ahvaz and Imam Khomeini hospital in Ramhormoz in
2015. Abuzar and Razi hospitals in Ahvaz were selected because they were the reference hospitals. In addition, Imam Khomeini hospital in Ramhormoz was selected because this city had the highest incidence of scorpion sting and snake bite in the province. This study aimed to assess direct medical and non-medical costs and indirect costs from a social perspective. The costs of snake bite and scorpion sting were analyzed in three parts as follows:

1) In the first part of the study, direct costs imposed on patients injured by scorpion sting and snake bite were identified and estimated.

2) The second part was devoted to the estimation of indirect costs.

3) In the third part of the study, data obtained from the previous two parts were combined and the average cost per patient was calculated. Then, in order to estimate the total economic burden in the country, the estimated average cost was multiplied by the total number of patients.

In this study, the incidence-based approach was used to obtain information about costs. In addition, bottomup approach was used to analyze the costs imposed on patients (14-16).

\subsection{Part I: Measurement of Direct Medical Costs}

In order to increase the accuracy of data collected on direct medical costs, we obtained the views of patients and professionals and simultaneously reviewed outpatient and inpatient medical records of people who were admitted to the studied hospitals from January 1 to December 31, 2015.

Because of the allocation of public subsidies to medications and hospital services, the actual costs are not reported fully; therefore, in this study we calculated the costs based on the prices set in the private sector (17).

\subsubsection{Calculation of Direct Medical Costs}

The average total direct medical costs per each patient with scorpion sting and snake bite were separately calculated as follows $(6,18-20)$ :

Total direct medical cost per patient $=$ (average number of visits $\times$ visit tariffs) + (average number of tests $\times$ tariffs for each test $)+$ (cost of every unit of medicine $\times$ number of medicine prescribed in a course of treatment) + (average number of hospitalization days $\times$ tariffs for each day of hospitalization $)+($ average number of diagnostic services $\times$ tariffs for each diagnostic service) + (average number of polyvalent serum $\times$ tariffs for each serum $)+($ average number of other services $\times$ tariffs for each course of service)

Moreover, in order to make international comparisons in this study, we converted the costs using purchasing power parity with an exchange rate of 11222.4 Rials to international dollars (21). 


\subsubsection{Measurement of Direct Non-Medical Costs}

In order to estimate this part of the costs, we used the patients' self-reports, which were collected through faceto-face or telephone interviews in the studied centers during the study period. Since a number of patients who were admitted to the reference treatment centers were living in places other than Ahvaz, we considered other costs such as travel costs, accommodation costs, and food costs as important components of direct non-medical costs.

\subsubsection{Calculation of Direct Non-Medical Costs}

The average total direct non-medical costs per patient with scorpion sting and snake bite were separately calculated as follows $(6,18-20)$ :

Average total direct non-medical costs per every patient $=$ Average number of visits to a health care center to receive medical services $\times$ cost of every visit $\times$ percentage of patients referring from other cities.

\subsection{Part 2: Measurement of Indirect Costs}

The data on costs were collected through face-to-face or telephone interviews with patients who received inpatient and outpatient services from the studied medical centers during the study period. In addition, informed consent was obtained from all patients.

\subsubsection{Calculation of Indirect Costs}

In order to calculate the potential productivity lost due to hospitalization and outpatient treatment, we used the human capital approach (20). In this study, the days when the patient rested at home due to disability was also counted as a part of hospitalization days.

Potential productivity lost due to hospitalization $=$ average number of days hospitalized $\times$ average wage

Potential productivity lost due to outpatient treatment $=$ average number of days that a patient spent on visiting medical center or receiving medication $\times$ average wage

According to the decree of the Ministry of Labor and Social Affairs, every eight hours of work is calculated as a working day.

In this study, we used GDP method to calculate the potential productivity lost due to premature mortality, the data on GDP per capita were obtained from the data published by the World Bank.

Potential productivity lost due to premature mortality was calculated using the following formula:

$$
\begin{aligned}
P L & =\sum_{i=1}^{N} \frac{W \times(1+g)^{i}}{(1+r)^{i}} \\
& \text { W }=\text { GDP per capita } \\
& \mathrm{g}=\text { economic growth rate } \\
\mathrm{I} & =\text { average number of years of life lost } \\
\mathrm{r} & =\text { discount rate }
\end{aligned}
$$

3.3. Part 3: Economic burden imposed on all patients injured by scorpion sting and snake bite

After estimating direct and indirect costs imposed on each patient with scorpion sting and snake bite and estimating the incidence of the injury in the country, the potential economic burden of scorpion sting and snake bite was calculated using the following formula:

Economic burden: Total cost (direct medical + direct non-medical + and indirect costs) $\times$ estimated number of patients injured by scorpion sting and snake bite in Iran

\subsubsection{Estimation of Incidence}

There is no written and regular report on the incidence of scorpion sting and snake bite in the country. However, a few studies and reports have examined and investigated this issue. National report on the registration of scorpion sting injuries in 2009 published by disease control center is one of the reports which cover the data from 41 universities of medical sciences. According to this report, the total number of cases of scorpion sting in 2009 was 44,366 cases and the incidence of scorpion sting in the whole country was 59.5 per 100,000 people. Khozestan province with an average incidence of 541 cases per 100,000 people accounts for more than half of the cases of scorpion sting in the country (22). Moreover, Dehghani et al. (2014) reviewed and analyzed the 10-year trend of snake bite in Iran from 2002 to 2011. The results showed that during the study period a total of 53,787 cases of snake bites were reported by medical centers in Iran. The annual incidence of snake bites ranged from 4.5 to 9.1 per 100,000 people during this decade and the number of deaths registered was 67 cases. Most cases of snake bites occurred in the South and Southwest provinces of Iran. The highest incidence of snake bites was 6231 cases reported in 2016 and the lowest incidence was 4696 cases reported in 2010 (23). Therefore, as the actual data on the incidence of scorpion sting and snake bite are not easily available, different sources were used to collect the related data.

Thus, according to the abovementioned data, the estimated average annual incidences of scorpion sting and snake bite in the country, respectively, are about 42497 and 5379 cases. In addition, of the total cases, 20 and 7 patients die of scorpion sting and snake bite, respectively.

\section{Results}

In this study, a total of 655 patients were studied. Of whom 404 patients were injured by scorpion sting and 251 by snake bite. Table 1 presents the results of descriptive study of patients injured by scorpion sting and snake bite classified by gender, education, employment status, living in urban or rural areas, and mean age of the patients. 
Table 1. Frequency Distribution of Patients Injured by Scorpion Sting and Snake Bite by Age, Gender, Level of Education, Employment Status, and Place of Residence

\begin{tabular}{|c|c|c|c|c|c|}
\hline Variables & Scorpion & Percentage & Snake & Percentage & Total Patients \\
\hline Total pateints & 404 & 61.8 & 251 & 38.2 & 655 \\
\hline Men & 174 & 52.5 & 157 & 47.5 & 331 \\
\hline Women & 230 & 71 & 94 & 29 & 324 \\
\hline Without university education & 247 & 61.8 & 153 & 38.2 & 400 \\
\hline With university education & 157 & 61.6 & 98 & 38.4 & 255 \\
\hline Employed & 219 & 60.7 & 142 & 39.3 & 361 \\
\hline Unemployed & 185 & 63 & 109 & 37 & 294 \\
\hline Urban residency & 172 & 67.2 & 84 & 32.8 & 256 \\
\hline Rural residency & 232 & 58.1 & 167 & 41.9 & 399 \\
\hline Average age & 30 & & 29 & & 29.33 \\
\hline
\end{tabular}

As Table 1 shows, the mean age of all patients was 29.33 years.

\subsection{Direct Medical and Non-Medical Costs}

Table 2 presents the costs of diagnostic and therapeutic services (direct medical costs) and the costs of other required services (direct non-medical costs) per patient injured by scorpion sting and snake bite.

Among the components of direct costs, hoteling costs accounted for the largest share of medical costs (51\% in patients injured by scorpion sting and $46 \%$ in patients injured by snake bite). Moreover, the cost of visits accounted for $15 \%$ of total costs spent by patients injured by scorpion sting and the cost of anti-venom polyvalent accounted for $18 \%$ of total costs spent by patients injured by snake bite. The lowest percentage of costs spent by scorpion sting patients was related to the costs spent on other services including pharmaceutical services management, blood transfusions or using blood products, and using ambulance. The lowest percentage of costs spent by snake bite patients was related to diagnostic services including radiotherapy, radiography, ultrasound, and then costs of using disposable goods including IV, tubing set, health bags, and oral thermometers.

\subsection{Direct Non-Medical Costs}

Some of the patients injured by scorpion sting and snake bite who are involved in critical conditions are usually forced to get health care services through visiting referral centers in other cities. Therefore, such a condition could considerably increase direct non-medical costs. Direct non-medical costs include transportation costs, accommodation costs, and meals costs. Direct non-medical costs spent by patients injured by scorpion sting and snake bite, respectively, are estimated as 130 PPP\$ and 150 PPP\$ per year. The largest share of these costs is related to the costs of accommodation of patients' accompanies both inside and outside the hospital.

\section{Indirect costs}

According to the results of our study, none of the patients injured by scorpion sting and snake bite lost their job due to treatment services or disease complications. As shown in Table 3, indirect costs per patient injured by scorpion sting and snake bite were estimated as 112 PPP\$ and 523 PPP\$ per year, respectively. The highest share of indirect costs was related to the cost of potential productivity lost due to hospitalization or death. In addition, the results showed that indirect costs accounted for a substantial part of the total costs, as it accounted for $25 \%$ of total costs spent by patients injured by snake bite.

\subsection{Estimation of the Total Cost per Every Patient Injured by Scorpion Sting and Snake Bite}

Figure 1 and Table 3 present a summary of direct medical and non-medical costs and indirect costs per each patient in the country. The results showed that direct medical costs were one of the main components of the total estimated cost of treating patients injured by scorpion sting and snake bite and accounted for about $68 \%$ to $80 \%$ of total costs of the disease (Figure 1).

\subsection{Economic Burden of Direct and Indirect Costs of Scorpion Sting and Snake Bite in Iran}

With regard to the estimated number of patients under treatment in a year, economic burden of scorpion sting and snake bite was calculated and the results are presented in Table 4. As shown in Table 4, the estimated total direct costs for patients injured by scorpion sting and snake bite 
Table 2. Average Direct Medical and Non-Medical Costs Per Patient Injured by Scorpion Sting and Snake Bite in $2015^{\mathrm{a}}$

\begin{tabular}{|c|c|c|c|c|}
\hline Service Type & Scorpion (PPPS) & Percentage & Snake (PPPS) & Percentage \\
\hline \multicolumn{5}{|l|}{ Direct medical cost } \\
\hline Visit & $140 \pm 28$ & 15 & $171 \pm 24$ & 12 \\
\hline Nursing services & $22 \pm 6$ & 2 & $39 \pm 2$ & 3 \\
\hline Laboratory & $54 \pm 7$ & 5 & $95 \pm 9$ & 7 \\
\hline Diagnosis services & $36 \pm 8$ & 4 & $19 \pm 8$ & 1 \\
\hline Medicine & $73 \pm 20$ & 8 & $120 \pm 16$ & 8 \\
\hline Hoteling & $487 \pm 203$ & 51 & $656 \pm 200$ & 46 \\
\hline Polyvalent anti-scorpion serum & 100 & 11 & 248 & 18 \\
\hline Consumer accecories & $30 \pm 8$ & 3 & $36 \pm 4$ & 2 \\
\hline Other services & $8 \pm 2$ & 1 & $47 \pm 5$ & 3 \\
\hline Total & 950 & 100 & 1431 & 100 \\
\hline \multicolumn{5}{|l|}{ Direct non-medical costs } \\
\hline Transportation & $35 \pm 10$ & 27 & $37 \pm 12$ & 25 \\
\hline Accomodation & $61 \pm 19$ & 47 & $75 \pm 27$ & 50 \\
\hline Meals & $34 \pm 6$ & 26 & $38 \pm 7$ & 25 \\
\hline Total & 130 & 100 & 150 & 100 \\
\hline Total cost & 1080 & & 1581 & \\
\hline
\end{tabular}

Abbreviations: PPP\$, purchasing power parity dollar.

${ }^{\mathrm{a}}$ Data presented as mean $\pm \mathrm{SD}$.

Table 3. Estimation of Average Direct Medical and Non-Medical Costs, Indirect Costs, Total Costs, and Ratio of Direct to Indirect Costs per Patient Injured by Scorpion Sting and Snake Bite in $2015^{\mathrm{a}, \mathrm{b}}$

\begin{tabular}{lccc}
\hline ID & COST & Scorpion Sting (PPP\$) & Snake Bite (PPP\$) \\
\hline $\mathbf{1}$ & DMC & $950 \pm 280(80)$ & $1431 \pm 270(68)$ \\
$\mathbf{2}$ & DNMC & $130 \pm 35(11)$ & $150 \pm 45(7)$ \\
$\mathbf{3}$ & IC & $112(9)$ & $523(25)$ \\
$\mathbf{4}$ & DC to IC Ratio & 9.6 & 3 \\
& Total Cost & $1192(100)$ & $2104(100)$ \\
\hline
\end{tabular}

Abbreviations: DC to IC Ratio, ratio of direct cost to indirect costs; DMC, direct medical cost; DNMC: direct non-medical cost; IC, indirect costs; and PPP\$, purchasing power parity dollar.

${ }^{a}$ Data presented as No. (\%).

${ }^{\mathrm{b}}$ Annual indirect costs caused by presenteeism and absenteeism based on human capital approach

in the country were 45,896,760 PPP \$ and 8,504,199 PPP \$, respectively. Moreover, the estimated total indirect costs for patients injured by scorpion sting and snake bite in the country were 4,759,664 PPP \$ and 2,813,217 PPP \$, respectively (Table 4). Finally, the total economic burden of scorpion sting and snake bite in the country was 50,656,424 PPP \$ and 11,317,416 PPP \$, respectively. The total burden was 61,973,840 PPP \$ (Table 4).

\section{Discussion}

The present study aimed to estimate the economic burden of scorpion sting and snake bite from a social perspective in Iran.

The results of this study showed that scorpion sting and snake bite could impose a significant economic burden on the health system and community. As mentioned above, no relevant study has been published so far; however, the results of our study are somewhat consistent 
Table 4. Estimation of Average Direct Medical and Non-Medical Costs, Indirect Costs, Total Costs, and Ratio of Direct to Indirect Costs Per Patient Injured by Scorpion Sting and Snake Bite in Iran in 2015

\begin{tabular}{|c|c|c|c|c|c|c|}
\hline Disease Type & Number of Patients in Iran & DMC, (PPP\$) & DNMC, (PPPS) & IC, (PPP\$) & COI, (PPPS) & DC to IC Ratio \\
\hline Scorpion sting & 42497 & $40,372,150$ & $5,524,610$ & $4,759,664$ & $50,656,424$ & 9.6 \\
\hline Snake bite & 5379 & $7,697,349$ & 806,850 & $2,813,217$ & $11,317,416$ & 3 \\
\hline Total & 47876 & $48,069,499$ & $6,331,460$ & $7,572,881$ & $61,973,840$ & 7.2 \\
\hline
\end{tabular}

Abbreviations: COI, cost of illness; DC to IC Ratio, ratio of direct cost to indirect costs; DMC, direct medical cost; DNMC: direct non-medical cost; IC, indirect costs; PPPS, purchasing power parity dollar.

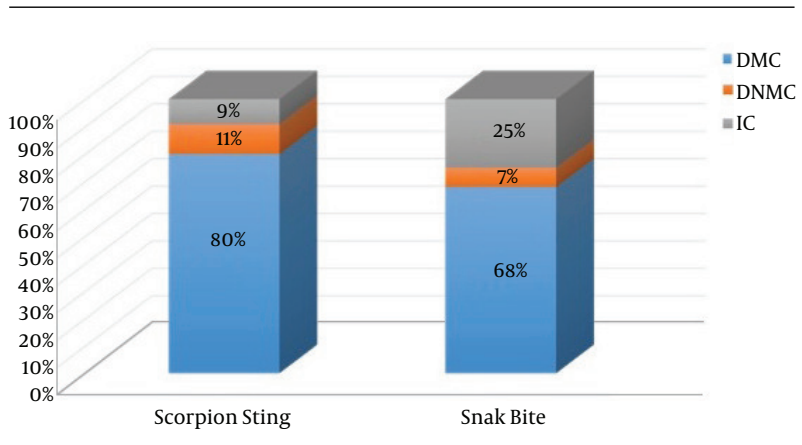

Figure 1. Direct Medical and Non-Medical Costs and Indirect Costs as a Percentage of the Total Cost for Each Group of Patients

with the results of Edward et al.'s study which reported the catastrophic costs of anti-venom serum and high economic burden of the disease (13). In addition, the results of the present study showed that the average total costs, which covered direct medical costs, direct non-medical costs, and indirect costs, per patient injured by scorpion sting and snake bite were 1192 PPP \$ and 2104 PPP \$, respectively. Direct medical costs had the largest share of the total cost of treating patients injured by scorpion sting and snake bite and accounted for about $68 \%$ to $80 \%$ of the total costs of the disease. In addition, the total annual economic burden for patients injured by scorpion sting and snake bite in the country was 50,656,424 PPP \$ and 11,317,416 PPP $\$$, respectively. The total burden was $61,973,840$ PPP \$.

In addition, the results showed that $11 \%$ and $18 \%$ of all direct medical costs were devoted to the purchase of antivenom polyvalent serum in scorpion sting and snake bite patients, respectively. Therefore, although the use of antivenom serum is very effective (3), because of the lack of appropriate native guides, there is a very high frequency of polyvalent serum injection to treat scorpion sting and snake bite in the country. On the other hand, as anti-venom polyvalent serum is available in the health centers free of charge, it could impose a huge direct economic burden on the health system.

This result is in line with the findings of Edward et al.'s study which reported that anti-venom serum is effective but expensive (13). Therefore, it might be helpful and cost effective to prepare an appropriate treatment protocol for such patients to utilize anti-venom serum more efficiently in a cost-saving manner.

In addition, the results showed that the cost of hoteling services accounted for the largest share of direct medical costs spent by patients injured by scorpion sting and snake bite. It might be due to the fact that this group of patients required more high-cost services in hospitals like intensive care unit services. It might be concluded that the lack of appropriate and timely treatment of patients might result in increasing the length of stay and the economic burden of the disease.

Moreover, the finding of this study showed that of all the patients injured by scorpion sting and snake bite $57.4 \%$ and $66.5 \%$ were living in rural areas, respectively. As the service centers for treating scorpion sting and snake bite are located in urban areas, it might impose a higher level of economic burden, especially in terms of direct nonmedical and indirect costs including costs of transportation, accommodation, meals, medical leave, time off, and productivity loss.

Furthermore, indirect costs spent by patients injured by scorpion sting and snake bite accounted for $25 \%$ and $9 \%$ of the total costs, respectively. The findings indicate that indirect costs are an important cost component; similar to direct costs, indirect costs may increase with the progression of the disease and thus may impose a high burden on the community. As a result, when calculating the economic burden of diseases, they might be also considered and included, as well.

On the other hand, the results showed that indirect costs imposed by snake bite are more than indirect costs imposed by scorpion sting.

Finally, the results of the study showed that with the development and progression of the disease, the patients will require more hospitalization services which can significantly increase the direct costs. Moreover, because of absenteeism and loss of productivity due to illness, there 
will be an increase in indirect costs, as well.

As mentioned in previous sections, there was no up-todate and comprehensive document on the exact number of scorpion stings and snake bites in the country and it was one of the limitations of this study. As a result, in this study we used sporadic reports and resources that had related data.

\subsection{Conclusions}

The results of this study showed that the economic burden of scorpion sting and snake bites on payers, patients, and the community is dramatically high; therefore, it is necessary to adopt proper preventive and medical activities to prevent the increase of the related economic burden.

\section{Acknowledgments}

The present article was extracted from the thesis written by Iman Mashhadi and was financially supported by Shiraz University of Medical Sciences grants No. 94-01-0710222. The authors would like to express their thanks to health deputy of Jondi Shapour University of Medical Sciences, Ahvaz, and the principals and staffs working at Razi and Abouzar hospitals in Ahvaz and Imam Khomeini hospital in Ramhormoz who helped us conduct this project and also the center for development of clinical research of Nemazee hospital and Dr. Nasrin Shokrpour for editorial assistance.

\section{Footnote}

Financial Disclosure: No conflict of interest was associated with this work.

\section{References}

1. Kalantari H, Davari M, Akbari M, Hejazi SM, Kalantari M, Zakerin $S$, et al. The estimation of direct medical costs of treating patients with chronic hepatitis B and C in iran. Int J Prev Med. 2012;3(3):191-6. [PubMed: 22448312].

2. Ravangard R, Hatam N, Teimourizad A, Jafari A. Factors affecting the technical efficiency of health systems: A case study of economic cooperation organization (ECO) countries. International J of Health Policy and Management. 2014;3(2):63-69.

3. Nassaji Jahromi N, Jafari A, Kavosi Z, Shokrpour N, Sajjadnia Z, Ravangard R. Demand for dental services in Shiraz, Iran, 2013. The Health Care Manager. 2016;35(4):340-349.
4. Bahadori M, Ravangard R, Alimohammadzadeh K, Hosseini SM. Plan and road map for health reform in Iran. BMJ. 2015;351:h4407.

5. Kavosi Z, Keshtkaran A, Hayati R, Ravangard R, Khammarnia M. Household financial contribution to the health System in Shiraz, Iran in 2012. International J of Health Policy and Management. 2014;3(5):243249. doi: 10.5812/hepatmon.15(4)2015.25854. [PubMed: 25977694].

6. Keshavarz K, Kebriaeezadeh A, Alavian SM, Akbari Sari A, Abedin Dorkoosh F, Keshvari M, et al. Economic burden of hepatitis B virusrelated diseases: evidence from iran. Hepat Mon. 2015;15(4):25854. doi: 10.5812/hepatmon.15(4)2015.25854. [PubMed: 25977694].

7. Mashayekhi M. Manual for controlling venomous animals bite. Tehran: Seda; 2006. pp. 9-14.

8. WHO . Rabies and envenomings. Report of a consultative meeting. Geneva: World Health Organization; 2007.

9. Shahi M. Study of scorpion fauna in regions with high incidence of scorpion sting in Hormozgan province, 2006-2007. Hormozgan Med J. 2008;12(4):207-14.

10. Saghafipour A. Epidemiologic evaluation of scorpion sting in Qom province during 2001-2011. J Safety Promotion and Injury Prevention. 2013;1(2):95-101.

11. Zamani Alavijeh F. Epidemiologic evaluation and estimation of 24month incidence of scorpion sting and snake bite in patients referring to 22 Bahman hospital in Masjed Soleiman based on time series model. J Safety Promotion Injury Prevention. 2013;1(4):190-7.

12. Shadnia S. Prevention and treatment of snake bites. Tehran: Seda; 2009. pp. 1-12.

13. Armstrong EP, Bakall M, Skrepnek GH, Boyer LV. Is scorpion antivenom cost-effective as marketed in the United States?. Toxicon. 2013;76:394-8. doi: 10.1016/j.toxicon.2013.09.001. [PubMed: 24055550].

14. Droodi R. Comprehensive review of methods for calculating economic burden of cancer. Hakim Res J. 2013;16(4):349-57.

15. Hu M, Chen W. Assessment of Total Economic Burden of Chronic Hepatitis B (CHB)-Related Diseases in Beijing and Guangzhou, China. Value in Health Regional Issues. 2009;12:89-92.

16. Harbarth S, Szucs T, Berger K, Jilg W. The economic burden of hepatitis B in Germany. Eur J Epidemiol. 2000;16(2):173-7. [PubMed: 10845268].

17. Haghparast-Bidgoli H, Kiadaliri AA, Skordis-Worrall J. Do economic evaluation studies inform effective healthcare resource allocation in Iran? A critical review of the literature. Cost EffResour Alloc. 2014;12:15. doi: 10.1186/1478-7547-12-15. [PubMed: 25050084].

18. Ong SC, Lim SG, Li SC. How big is the financial burden of hepatitis B to society? A cost-of-illness study of hepatitis B infection in Singapore. J Viral Hepat. 2009;16(1):53-63. doi: 10.1111/j.1365-2893.2008.01042.x. [PubMed: 19192158]

19. Tu HAT, Woerdenbag HJ, Riewpaiboon A, Kane S, Le DM, Postma MJ. Cost of Illness of Chronic Hepatitis B Infection in Vietnam. Value in Health Regional Issues. 2012;1(1):23-8.

20. Kavosi Z, Zare F, Jafari A, Fattahi MR. Economic burden of hepatitis B virus infection in different stages of disease; a report from southern iran. Middle East J Dig Dis. 2014;6(3):156-61. [PubMed: 25093064].

21. Wold Bank. . PPP conversion factor, Private consumption (LCU per international \$) Available from: http://data.worldbank.org.

22. Country Reports Management Center of scorpion stinging incidents recorded in 2009. Available from: http://www.health.gov.ir/ncdc/ injury/SitePages/Home.aspx.

23. Dehghani R, Fathi B, Shahi MP, Jazayeri M. Ten years of snakebites in Iran. Toxicon. 2014;90:291-8. doi: 10.1016/j.toxicon.2014.08.063. [PubMed: 25193748]. 